\title{
Endovascular Therapy for Stroke Due to Basilar-Artery Occlusion
}

\author{
L.C.M. Langezaal, E.J.R.J. van der Hoeven, F.J.A. Mont'Alverne, J.J.F. de Carvalho, \\ F.O. Lima, D.W.J. Dippel, A. van der Lugt, R.T.H. Lo, J. Boiten, \\ G.J. Lycklama à Nijeholt, J. Staals, W.H. van Zwam, P.J. Nederkoorn, \\ C.B.L.M. Majoie, J.C. Gerber, M. Mazighi, M. Piotin, A. Zini, S. Vallone, \\ J. Hofmeijer, S.O. Martins, C.H. Nolte, K. Szabo, F.A. Dias, D.G. Abud, \\ M.J.H. Wermer, M.J.M. Remmers, H. Schneider, C.M. Rueckert, K.F. de Laat, \\ A.J. Yoo, P.-J. van Doormaal, A.C.G.M. van Es, B.J. Emmer, P. Michel, V. Puetz, \\ H.J. Audebert, O.M. Pontes-Neto, J.-A. Vos, L.J. Kappelle, A. Algra, \\ and W.J. Schonewille, for the BASICS Study Group*
}

\section{A BSTRACT}

The authors' full names, academic degrees, and affiliations are listed in the Appendix. Address reprint requests to Dr. Schonewille at the St. Antonius Hospital, Koekoekslaan 1, 3435 CM, Nieuwegein, the Netherlands, or at w.schonewille@ antoniusziekenhuis.nl.

*A complete list of the members of the BASICS Study Group is provided in the Supplementary Appendix, available at NEJM.org.

Drs. Langezaal and van der Hoeven contributed equally to this article.

N Engl J Med 2021;384:1910-20. DOI: 10.1056/NEJMoa2030297

Copyright (c) 2021 Massachusetts Medical Society.

\section{BACKGROUND}

The effectiveness of endovascular therapy in patients with stroke caused by basilarartery occlusion has not been well studied.

METHODS

We randomly assigned patients within 6 hours after the estimated time of onset of a stroke due to basilar-artery occlusion, in a 1:1 ratio, to receive endovascular therapy or standard medical care. The primary outcome was a favorable functional outcome, defined as a score of 0 to 3 on the modified Rankin scale (range, 0 to 6, with 0 indicating no disability, 3 indicating moderate disability, and 6 indicating death) at 90 days. The primary safety outcomes were symptomatic intracranial hemorrhage within 3 days after the initiation of treatment and mortality at 90 days.

RESULTS

A total of 300 patients were enrolled (154 in the endovascular therapy group and 146 in the medical care group). Intravenous thrombolysis was used in $78.6 \%$ of the patients in the endovascular group and in $79.5 \%$ of those in the medical group. Endovascular treatment was initiated at a median of 4.4 hours after stroke onset. A favorable functional outcome occurred in 68 of 154 patients (44.2\%) in the endovascular group and 55 of 146 patients (37.7\%) in the medical care group (risk ratio, 1.18; $95 \%$ confidence interval [CI], 0.92 to 1.50). Symptomatic intracranial hemorrhage occurred in $4.5 \%$ of the patients after endovascular therapy and in $0.7 \%$ of those after medical therapy (risk ratio, 6.9; 95\% CI, 0.9 to 53.0); mortality at 90 days was $38.3 \%$ and $43.2 \%$, respectively (risk ratio, 0.87 ; $95 \%$ CI, 0.68 to 1.12 ).

\section{CONCLUSIONS}

Among patients with stroke from basilar-artery occlusion, endovascular therapy and medical therapy did not differ significantly with respect to a favorable functional outcome, but, as reflected by the wide confidence interval for the primary outcome, the results of this trial may not exclude a substantial benefit of endovascular therapy. Larger trials are needed to determine the efficacy and safety of endovascular therapy for basilar-artery occlusion. (Funded by the Dutch Heart Foundation and others; BASICS ClinicalTrials.gov number, NCT01717755; Netherlands Trial Register number, NL2500.) 
B ASILAR-ARTERY OCCLUSION, WHICH ACcounts for approximately $10 \%$ of all ischemic strokes caused by intracranial proximal large-vessel occlusion, is associated with high morbidity and mortality. ${ }^{1,2}$ Case reports of favorable clinical outcomes associated with endovascular therapy for basilar-artery occlusion have impeded recruitment in randomized, controlled trials. ${ }^{3,4}$ One trial was terminated prematurely because of deficient recruitment..$^{5}$ In a metaanalysis of case series of patients with basilarartery occlusion, published between 1988 and $2004,{ }^{6}$ and in a prospective registry (Basilar Artery International Cooperation Study [BASICS]), for which data were collected between 2002 and 2007 , the majority of patients underwent endovascular therapy. ${ }^{7}$ Neither suggested a benefit of endovascular therapy over intravenous thrombolysis.

In a subgroup analysis of the BASICS registry, patients with severe neurologic deficits or a National Institutes of Health Stroke Scale (NIHSS) score higher than 19 (range, 0 to 42, with higher scores indicating worse deficits) may have had a lower risk of a poor outcome with endovascular therapy than with antithrombotic therapy. However, no definite conclusions could be drawn. ${ }^{7}$

Few patients with basilar-artery occlusion have been included in major trials of endovascular therapy, which have mainly enrolled patients with strokes in the anterior cerebral circulation. The vascular anatomy, clinical presentation, and severity of neurologic deficit in patients with basilar-artery occlusion differ from those in patients with anterior-circulation strokes. These differences suggest that trials of endovascular treatment should be conducted independently for patients with strokes in the basilar-artery territory.

We conducted a randomized trial involving patients with strokes due to basilar-artery occlusion. Our trial was conducted from 2011 through 2019, although evidence published in 2015 showed efficacy of endovascular therapy in anterior-circulation large-vessel occlusion. ${ }^{8}$ We based the current trial on information acquired from the BASICS registry. We compared the efficacy and safety of endovascular therapy initiated within 6 hours after the estimated time of basilar-artery occlusion with those of medical therapy.
METHODS

\section{TRIAL DESIGN}

We conducted a multicenter, open-label, international, randomized, controlled trial with blinded outcome assessment. The trial design has been published previously. ${ }^{9}$ The trial protocol and amendments, available with the full text of this article at NEJM.org, were approved by the national regulatory authority in each participating country and by the institutional review board at each participating center.

Eligible patients or their legally authorized representatives provided written informed consent before enrollment. If required by national laws or regulations for obtaining consent, after consultation with the investigator, an independent physician who was not otherwise participating in the trial provided consent. Members of the executive committee designed and oversaw the trial and made the decision to submit the manuscript for publication. The first draft of the manuscript was written by the first and second authors; the penultimate author analyzed the data. All the authors vouch for the accuracy and completeness of the presented data, for adherence of the trial to the protocol, and for the accurate reporting of adverse events. The trial was monitored by an independent data and safety monitoring board. Decisions related to safety and trial continuation were made by the executive committee on the basis of recommendations by the data and safety monitoring board.

\section{PATIENTS AND PARTICIPATING CENTERS}

The trial was performed in 23 centers in seven countries, which are listed in the Supplementary Appendix, available at NEJM.org. In the initial plan for the trial, patients were eligible for inclusion if they were younger than 85 years of age and had acute symptoms and signs compatible with ischemia in the basilar-artery territory, a proven basilar-artery occlusion on computed tomographic (CT) angiography (CTA) or magnetic resonance angiography (MRA), and an NIHSS score of 10 or more. During the trial enrollment period (from October 23, 2011, through December 6, 2019), participating centers entered into a screening log all patients with acute basilarartery occlusion who were eligible to participate in the trial but had not undergone randomization (Table S5 in the Supplementary 
Appendix) and all those who were not eligible to participate.

Because of slow enrollment and data from the screening log suggesting uncertainty among enrolling physicians about the best treatment approach in certain subgroups of patients, 4 years into the trial, after 91 patients had undergone randomization, the inclusion criteria were expanded to allow enrollment of patients who were 85 years of age or older, those who had an NIHSS score of less than 10, and those who had contraindications to intravenous thrombolysis (Table S6).

Basilar-artery occlusion was defined as a complete obstruction of flow in any portion of the artery. The estimated time of basilar-artery occlusion was defined as the time of onset of acute symptoms leading to the clinical diagnosis of basilar-artery occlusion or, if not known, the last time the patient's condition was observed to be normal before the onset of stroke symptoms; these times were determined by the physician who enrolled the patient in the trial. ${ }^{7}$ Endovascular therapy had to be feasible within 6 hours and intravenous thrombolysis in patients assigned to the endovascular therapy group had to be initiated within 4.5 hours after the estimated time of basilar-artery occlusion. Patients with intracranial hemorrhage; extensive, bilateral brainstem infarction on CT; cerebellar mass effect; or acute hydrocephalus evident on neuroimaging were excluded. Detailed inclusion and exclusion criteria are provided in the Supplementary Appendix.

\section{RANDOMIZATION AND TREATMENTS}

Patients were randomly assigned in a 1:1 ratio to receive endovascular therapy or standard medical care. Randomization was conducted with the use of a central, Web-based procedure with permuted blocks (size 2) and was stratified according to center, use of intravenous thrombolysis, and NIHSS score $(<20$ or $\geq 20)$.

Medical care, which consisted of conventional care according to local protocols and national guidelines as determined by the site investigators, could include intravenous thrombolysis. The methods and devices used for endovascular thrombectomy were determined by the local interventional operator. Angioplasty or stenting of the vertebral artery in patients assigned to endovascular therapy was allowed if stenosis ham- pered access to the basilar artery, and stenting of the basilar artery was allowed if there was residual stenosis after thrombectomy. To establish patency of the basilar artery, interventionalists were asked to achieve visible outflow of contrast medium in at least one posterior cerebral artery after injection of the medium into a proximal vessel.

\section{OUTCOMES AND SAFETY}

The primary outcome was a favorable functional outcome, defined as a score of 0 to 3 on the modified Rankin scale (range, 0 to 6 , with 0 indicating no disability, 3 indicating moderate disability, and 6 indicating death) at 90 days. The primary safety outcomes were symptomatic intracranial hemorrhage detected on neuroimaging within 3 days after the initiation of treatment and mortality at 90 days. In the absence of an alternative explanation for clinical deterioration, symptomatic intracranial hemorrhage was defined according to the Heidelberg Bleeding Classification (hemorrhagic transformation of infarcted brain tissue, intracerebral hemorrhage both within and outside infarcted brain tissue, intracerebral hemorrhage outside the infarcted brain tissue, or intracranial-extracerebral hemorrhage and an increase of 4 points or more in the NIHSS score or an increase of 2 points or more in 1 of the 11 NIHSS subcategories). ${ }^{10}$

Secondary clinical outcomes were an excellent outcome, defined as a modified Rankin scale score of 0 to 2 (with 2 indicating slight disability); the NIHSS score at 24 hours; the distribution of scores on the modified Rankin scale; and health-related quality of life as measured on the European Quality of Life-5 Dimensions (EQ-5D) questionnaire at 90 days. The EQ-5D includes both the visual analogue scale component of overall health perception (range, 0 to 100, with higher scores indicating the patient's better perception of health) and the descriptive system yielding an index value (range, -0.33 to 1.00 , with higher values indicating a better health status). Secondary imaging outcomes were the extent of cerebral infarction - as measured with the posterior circulation Acute Stroke Prognosis Early CT Score (PC-ASPECTS, a 10-point grading system in which 1 or 2 points are deducted from 10 for each of the eight regions that may be infarcted) on noncontrast CT and CTA source images 
- and the basilar-artery patency, which is assessed on CTA or MRA at 24 hours ( \pm 6 hours). ${ }^{11}$

Follow-up of patients (to determine scores on the modified Rankin scale and EQ-5D questionnaire) was performed in a structured in-person or telephone interview at 90 days by an investigator who was unaware of the treatment group assignments. Clinical assessment of the NIHSS score was performed at baseline and at 24 hours after randomization by the local investigator, who was aware of the treatment group assignments. Baseline and follow-up images were independently assessed at a core laboratory by six neuroradiologists (one who interpreted all the images and one of five others who performed a second reading). An independent neuroradiologist arbitrated in case of disagreement.

\section{STATISTICAL ANALYSIS}

On the basis of the BASICS registry, we assumed that a favorable outcome would occur in $40 \%$ of the patients in the endovascular therapy group and in $30 \%$ of those in the medical care group, corresponding to an odds ratio of 1.56 and a risk ratio of 1.33. ' With a type I error rate (alpha) of $5 \%$ and a type II error rate (beta) of $20 \%$, we calculated that 712 patients would be required. To allow for the estimated dropout rate, we rounded this number to 750 . The publication of trial results on endovascular therapy in the anterior circulation and the slower-than-expected recruitment prompted us to reevaluate these assumptions. ${ }^{12-16}$ On the basis of the results of these trials, we calculated an anticipated pooled odds ratio of 2.17 and a pooled risk ratio of 1.43 for our primary outcome. In consensus with the data and safety monitoring board, we adapted our assumptions on sample size and anticipated a favorable outcome in $46 \%$ of the patients in the endovascular therapy group while maintaining the assumption of a favorable outcome in $30 \%$ of the patients in the medical care group, equivalent to an odds ratio of 2.0 and a risk ratio of 1.53. With an alpha level of $5 \%$ and a beta level of $20 \%$, the revised assumptions resulted in a required sample size of 282, which we rounded to 300 to account for dropouts.

The data and safety monitoring board evaluated the efficacy and safety of the trial with a triangular test (see the Supplementary Appendix) and recommended continuation of the trial after each of the blinded interim analyses. At the last interim analysis, on the basis of available assessment data from 90 days of follow-up of 252 patients who had undergone randomization, the board recommended that the trial continue to completion.

All the analyses were based on the intentionto-treat principle. We also planned an on-treatment analysis in which we excluded the patients who crossed over to the other treatment and an as-treated analysis according to the treatment that patients actually received. The statistical analysis plan is available at NEJM.org. In the two groups, we compared the dichotomous outcomes (a modified Rankin scale score of 0 to 3 and a modified Rankin scale score of 4 to 6) in terms of risk ratio. ${ }^{17}$ For the comparison of the two treatment groups with respect to the secondary outcomes of the NIHSS score and PC-ASPECTS at 24 hours and the EQ-5D at 90 days, we used linear regression and calculated the mean difference. Proportionality of the slope coefficients of categories of the modified Rankin scale score was affirmed by a test of parallel lines, which yielded a $P$ value of 0.70 .

There was no plan for adjustment of confidence intervals for multiple comparisons, and no conclusions can be drawn from these secondary outcome data. We prespecified calculation of adjusted effect estimates with multivariable analysis for randomization stratification factors and factors for which there was important baseline incomparability between the two treatment groups. There was no prespecified plan for handling missing data (although no patients were lost to follow-up at day 90, and no primary outcome data were missing).

Subgroup analyses were prespecified for the primary outcome according to the severity of deficit (mild, NIHSS score <10; moderate, 10 to 19; and severe, >19) and according to treatment with intravenous thrombolysis $(\leq 4.5$ hours after first symptom onset, $>4.5$ hours after first symptom onset, or no intravenous thrombolysis). In the endovascular therapy group, we compared the outcome of treatment less than 6 hours after the first symptom onset with that at or beyond 6 hours after the first symptom onset. These results are presented in a descriptive manner only because the trial was not powered for analyses of subgroups. 
RESULTS

PATIENT CHARACTERISTICS

The screening log showed that 300 of 424 eligible patients $(70.8 \%)$ were randomly assigned to a trial group. Of the 124 eligible patients who received treatment outside the trial, 98 (79.0\%) underwent endovascular therapy. These patients were similar in age (mean, 66.8 years) and sex ( $38 \%$ were women) to the patients who underwent randomization. Other characteristics of the eligible patients who did not undergo randomization were not recorded. The most frequent reasons for not enrolling potentially eligible patients were unavailability of the trial team (39 patients), transfer from another hospital with a specific request for endovascular treatment (14 patients), and the opinion on the part of the treatment team that there was lack of equipoise regarding the appropriate treatment of a particular patient (10 patients) and contraindication to intravenous thrombolysis (10 patients).

From October 2011 through December 2019, a total of 154 patients were randomly assigned to the endovascular group and 146 were randomly assigned to the medical treatment group. Crossover from one treatment group to the other occurred in 3 of 154 (1.9\%) patients assigned to the endovascular group and 7 of 146 (4.8\%) patients assigned to the medical group. No patients had been lost to follow-up at day 90 (Fig. S2). There was an imbalance in the incidence of atrial fibrillation (in $28.6 \%$ of the patients in the endovascular group and $15.1 \%$ of those in the medical care group); other baseline characteristics were similar in the two groups (Table 1). In the endovascular and medical care groups, 121 of 154 patients (78.6\%) and 116 of 146 patients (79.5\%), respectively, received intravenous thrombolysis. Second- and third-generation thrombectomy devices were used in $94 \%$ of the 154 patients who received endovascular therapy, and their use did not differ over the period of the trial (Table S4).

\section{EFFICACY OUTCOMES}

In the intention-to-treat analysis, 68 of 154 patients $(44.2 \%)$ in the endovascular treatment group had a favorable functional outcome (modified Rankin scale score, 0 to 3 at 90 days), as compared with 55 of 146 patients (37.7\%) in the medical treatment group (risk ratio, 1.18; 95\% confidence interval $[\mathrm{CI}], 0.92$ to $1.50 ; \mathrm{P}=0.19$ )
(Table 2). Figure 1 shows the distribution of the modified Rankin scale scores at 90 days in the two treatment groups; the baseline-adjusted common odds ratio across modified Rankin scale scores for endovascular therapy as compared with medical care was 1.35 (95\% CI, 0.88 to $2.88)$. A total of 54 patients (35.1\%) in the endovascular group and 44 patients (30.1\%) in the medical treatment group had a modified Rankin scale score of 0 to 2 (excellent outcome) (risk ratio, 1.17; $95 \%$ CI, 0.87 to 1.57 ). A total of $72 \%$ of the patients in the endovascular group had successful postprocedural reperfusion (a score of $2 \mathrm{~b}$ or 3 on the modified Thrombolysis in Cerebral Infarction scale, which ranges from 0 [no reperfusion] to 3 [full reperfusion in the distribution of the occluded artery]). Patency of the basilar artery at 24 hours, assessed by means of CTA, was $84.5 \%$ in the endovascular group and $56.3 \%$ in the medical group (Table 2). The results of other secondary outcomes are shown in Table 2; because there was no prespecified plan for adjustment of confidence intervals for multiple comparisons, no definite conclusions can be drawn from these data. On-treatment and as-treated analyses for the primary outcome showed results that were similar to those in the intention-to-treat analysis (Table S3).

\section{SAFETY OUTCOMES}

Mortality at 90 days was $38.3 \%$ in the endovascular group and $43.2 \%$ in the medical care group (risk ratio, $0.87 ; 95 \% \mathrm{CI}, 0.68$ to $1.12 ; \mathrm{P}=0.29$ ); the corresponding time-to-event curves are shown in Figure S3. The risk of symptomatic intracranial hemorrhage within 3 days after the initiation of treatment was $4.5 \%$ in the endovascular group and $0.7 \%$ in the medical care group (risk ratio, 6.9; 95\% CI, 0.9 to 53.0; $\mathrm{P}=0.06$ ) (Table 3). Malignant brain edema occurred in 17 patients $(11.0 \%)$ in the endovascular therapy group and in 7 patients $(4.8 \%)$ in the medical care group (risk ratio, 2.31; 95\% CI, 0.95 to $5.62 ; \mathrm{P}=0.06$ ).

\section{SUBGROUP ANALYSIS}

At 90 days after randomization, 20 of 31 patients $(65 \%)$ in the endovascular therapy group and 24 of 30 patients $(80 \%)$ in the medical care group had a mild deficit (NIHSS score $<10$ ) and a modified Rankin scale score of 0 to 3; a total of 31 of 42 patients (74\%) and 17 of 36 patients (47\%), respectively, had a moderate deficit (NIHSS score of 10 to 19) and favorable outcomes; and 17 of 


\begin{tabular}{|c|c|c|}
\hline Characteristic & $\begin{array}{l}\text { Endovascular Therapy } \\
\qquad(\mathrm{N}=154)\end{array}$ & $\begin{array}{l}\text { Medical Care } \\
(\mathrm{N}=146)\end{array}$ \\
\hline Age $-y r$ & $66.8 \pm 13.1$ & $67.2 \pm 11.9$ \\
\hline Female sex - no. (\%) & $54(35.1)$ & $50(34.2)$ \\
\hline \multicolumn{3}{|l|}{ Clinical history — no./total no. (\%) } \\
\hline Posterior-circulation stroke & $11 / 154(7.1)$ & $7 / 146(4.8)$ \\
\hline Hypertension & $93 / 154(60.4)$ & $82 / 145(56.6)$ \\
\hline Diabetes mellitus & $34 / 153(22.2)$ & $31 / 146(21.2)$ \\
\hline Atrial fibrillation or atrial fibrillation on 12-lead ECG & $44 / 154(28.6)$ & $22 / 146(15.1)$ \\
\hline \multicolumn{3}{|l|}{ Prestroke modified Rankin scale score — no./total no. (\%) $\dagger$} \\
\hline 0 & $123 / 153(80.4)$ & $112 / 146(76.7)$ \\
\hline 1 & $12 / 153(7.8)$ & $15 / 146(10.3)$ \\
\hline 2 & $15 / 153(9.8)$ & $17 / 146(11.6)$ \\
\hline 3 & $3 / 153(2.0)$ & $2 / 146(1.4)$ \\
\hline Treatment with intravenous thrombolysis — no. (\%) & $121(78.6)$ & $116(79.5)$ \\
\hline \multicolumn{3}{|l|}{ Time from stroke onset to intravenous thrombolysis - hrt } \\
\hline Median & 2.0 & 2.3 \\
\hline Interquartile range & $1.4-3.3$ & $1.6-3.5$ \\
\hline \multicolumn{3}{|l|}{ Time from stroke onset to endovascular therapy — hr $\int$} \\
\hline Median & 4.4 & NA \\
\hline Interquartile range & $3.3-6.2$ & NA \\
\hline \multicolumn{3}{|l|}{ NIHSS scoreq } \\
\hline Mean & 21.9 & 22.1 \\
\hline Median & 21 & 22 \\
\hline $\begin{array}{l}\text { Reperfusion on digital subtraction angiography, modified } \mathrm{TICl} \\
\qquad 2 \mathrm{~b} \text { or } 3-\text { no./total no. (\%)\| }\end{array}$ & $63 / 88(72)$ & NA \\
\hline
\end{tabular}

* Plus-minus values are means \pm SD. ECG denotes electrocardiography, and NA not applicable.

$\uparrow$ Scores on the modified Rankin scale range from 0 to 6 , with 0 indicating no disability, 3 indicating moderate disability, and 6 indicating death.

$\$$ Data on the time from stroke onset to intravenous thrombolysis were available for 116 patients in the endovascular therapy group and 113 patients in the medical care group.

$\int$ Data on the time from stroke onset to endovascular therapy were available for 145 patients in the endovascular therapy group and no patients in the medical care group.

I Scores on the National Institutes of Health Stroke Scale (NIHSS) range from 0 to 42, with higher scores indicating worse deficits.

|| The modified Thrombolysis in Cerebral Infarction ( $\mathrm{TICl}$ ) scale ranges from 0 (no reperfusion) to 3 (full reperfusion in the distribution of the occluded artery).

81 patients (21\%) and 14 of 80 patients (18\%), respectively, had a severe deficit (NIHSS score >19) and a favorable outcome (Fig. 2, and Table S2). These results are descriptive because the trial was not powered to make statistical comparisons among subgroups.

\section{DISCUSSION}

In our trial, there was not a statistically significant difference in the primary outcome between the group of patients who received endovascular therapy for basilar-artery occlusion initiated within 6 hours after the time of stroke and those who received medical therapy. The upper limit of the $95 \%$ confidence interval of the risk ratio of 1.50 cannot exclude the possibility of benefit from endovascular therapy if the original power calculation estimate of a 10 percentage-point difference for the primary outcome is considered to be clinically meaningful, but this possibility is excluded if the revised estimate of a 16 percent- 


\begin{tabular}{|c|c|c|c|}
\hline Outcome & $\begin{array}{c}\text { Endovascular } \\
\text { Therapy } \\
(\mathrm{N}=154)\end{array}$ & $\begin{array}{l}\text { Medical } \\
\text { Care } \\
(\mathrm{N}=146)\end{array}$ & $\begin{array}{c}\text { Risk Ratio, } \\
\text { Common Odds Ratio, } \\
\text { or Mean Difference } \\
(95 \% \mathrm{Cl}) 广 \overline{1}\end{array}$ \\
\hline $\begin{array}{l}\text { Primary outcome: favorable functional outcome at day 90: modified } \\
\text { Rankin scale score } \leq 3-\text { no. }(\%) \div\end{array}$ & $68(44.2)$ & $55(37.7)$ & $1.18(0.92$ to 1.50$) \mathbb{\int}$ \\
\hline \multicolumn{4}{|l|}{ Secondary clinical outcomes } \\
\hline Modified Rankin scale score at day 90 & & & $1.35(0.88$ to 2.88$) 9$ \\
\hline 0 & $8(5.2)$ & $6(4.1)$ & $1.17(0.44$ to 3.09$) \int$ \\
\hline $0-1$ & $27(17.5)$ & $19(13.0)$ & $1.32(0.79$ to 2.19$) \int$ \\
\hline $0-2 \|$ & $54(35.1)$ & $44(30.1)$ & $1.17(0.87$ to 1.57$) \int$ \\
\hline $0-4$ & $78(50.6)$ & $71(48.6)$ & $1.05(0.85$ to 1.29$) \int$ \\
\hline $0-5$ & $95(61.7)$ & $83(56.8)$ & $1.09(0.92$ to 1.29$) \int$ \\
\hline NIHSS score at $24 \mathrm{hr}$ - median (interquartile range) ${ }^{* * *}$ & $11.0(3.0-37.5)$ & $15.0(5.0-36.5)$ & $-0.79(-3.90$ to 2.31$) \dagger$ \\
\hline \multicolumn{4}{|l|}{ EQ-5D questionnairetr } \\
\hline Visual analogue scale at day 90 & $67.6 \pm 21.3$ & $61.9 \pm 24.8$ & $6.0(-1.9$ to 13.8$) \dagger \dagger$ \\
\hline Index value at day 90 & $0.65 \pm 0.32$ & $0.61 \pm 0.32$ & $0.05(-0.05$ to 0.16$) \uparrow$ \\
\hline \multicolumn{4}{|l|}{ Secondary imaging outcomes } \\
\hline Patency at $24 \mathrm{hr}$ CT angiography — no./total no. (\%) $\int \mathbb{\int}$ & $93 / 110(84.5)$ & $54 / 96(56.3)$ & $1.43(1.18$ to 1.74$) \int$ \\
\hline \multicolumn{4}{|l|}{ PC-ASPECTS at $24 \mathrm{hr}$ — median (interquartile range) $१ \rrbracket$} \\
\hline Noncontrast CT & $8.0(6.0-9.0)$ & $8.0(6.0-9.0)$ & $-0.03(-0.66$ to 0.59$) \dagger \dagger$ \\
\hline CT angiography & $9.0(8.0-10.0)$ & $9.0(7.0-10.0)$ & $0.32(-0.24$ to 0.88$) \dagger$ \\
\hline Primary safety outcome: overall mortality at day $90-$ no. (\%) & $59(38.3)$ & $63(43.2)$ & $0.87(0.68$ to 1.12$) \int$ \\
\hline
\end{tabular}

* Plus-minus values are means \pm SD. CT denotes computed tomography.

$\dagger$ Estimates were adjusted for intravenous thrombolysis, NIHSS score, and atrial fibrillation. The $95 \%$ confidence intervals were not adjusted for multiple comparisons.

$\mp$ Scores on the modified Rankin scale of functional disability range from 0 to 6 , with 0 indicating no disability, 3 indicating moderate disability, and 6 indicating death. A score of 2 or less indicates an excellent outcome (functional independence). $P=0.19$ for the comparison of the risk ratio values.

$\int$ The effect measure was expressed as a risk ratio.

9 The effect measure was expressed as a common odds ratio.

Among the modified Rankin scale scores at day 90 , only a score of 0 to 2 was a prespecified secondary outcome.

** Data on the NIHSS score at 24 hours were available for surviving patients (145 in the endovascular therapy group and 129 in the medical care group). Data were missing for 9 patients.

$\dagger \dagger$ The effect measure was expressed as a mean difference.

Two components of the European Quality of Life-5 Dimensions (EQ-5D) questionnaire are shown. On the visual analogue scale component of overall health perception, the range is 0 to 100, with higher scores indicating the patient's better perception of health. Data on the EQ-5D visual analogue scale at day 90 were available for 72 patients in the endovascular therapy group and 59 patients in the medical care group. On the descriptive system yielding an index value, the range is -0.33 to 1.00 , with higher values indicating better health status Data on the EQ-5D index value at day 90 were available for 78 patients in the endovascular therapy group and 65 patients in the medical care group.

If Patency was defined as a score of 2 or 3 on the modified Arterial Occlusive Lesion scale, which ranges from 0 (complete occlusion) to 3 (complete recanalization and restoration of the target artery). Two patients in the endovascular therapy group and 3 patients in the medical care group had a modified arterial occlusive lesion.

9 1 The posterior circulation Acute Stroke Prognosis Early CT Score (PC-ASPECTS) is a 10-point grading system in which 1 or 2 points are deducted from 10 for each of the eight regions that may be infarcted. Data on PC-ASPECTS on noncontrast CT were available for 129 patients in the endovascular therapy group and 115 patients in the medical care group. Data on PC-ASPECTS on CT angiography were available for 110 patients in the endovascular therapy group and 98 patients in the medical care group.

age-point difference is used. The common odds ratio across modified Rankin scale scores indicated no significant between-group difference.
The trial therefore did not show an advantage of endovascular therapy over medical therapy, but these findings may be inconclusive. These re- 
sults may inform the design of future trials in this field, for which recruitment and implementation have been difficult. Secondary outcomes did not differ significantly between the treatment groups with respect to excellent outcome, mortality, and some other secondary outcomes.

In keeping with previous trials involving patients with an anticipated poor prognosis from stroke, such as hemicraniectomy trials for brain swelling after hemispheric infarction, we defined a favorable outcome as a modified Rankin scale score of 0 to $3 .{ }^{18}$ In our trial, the incidence of a favorable outcome (38\%) in the medical care group was higher than anticipated for patients with a basilar-artery stroke.

The percentage of patients in the medical group in our trial who had symptomatic intracranial hemorrhage was lower than that in trials of endovascular treatment of anterior-circulation strokes. ${ }^{8}$ The incidence of basilar-artery patency at 24 hours in the medical group in our trial was also higher than that in MR CLEAN (Multicenter Randomized Clinical Trial of Endovascular Treatment for Acute Ischemic Stroke in the Netherlands), a large trial of endovascular treatment for patients with anterior-circulation stroke. ${ }^{12}$ Intravenous thrombolysis may be more successful in opening the vessel in basilar-artery occlusion than in anterior-circulation vessel occlusion, but direct comparisons between these trials cannot be made.

A previous randomized trial of endovascular treatment of basilar-artery occlusion, the Endo-

\begin{tabular}{|c|c|c|}
\hline Variable & $\begin{array}{l}\text { Endovascular } \\
\text { Therapy } \\
(N=154)\end{array}$ & $\begin{array}{l}\text { Medical } \\
\text { Care } \\
(\mathrm{N}=146)\end{array}$ \\
\hline \multicolumn{3}{|l|}{ Serious adverse events - no. (\%) } \\
\hline 0 & $82(53.2)$ & $95(65.1)$ \\
\hline 1 & $48(31.2)$ & $33(22.6)$ \\
\hline$>1$ & $24(15.6)$ & $18(12.3)$ \\
\hline $\begin{array}{c}\text { Symptomatic intracranial hemorrhage } \\
\leq 3 \text { days after initiation of } \\
\text { treatment }- \text { no. }(\%)\end{array}$ & $7(4.5)$ & $1(0.7)$ \\
\hline \multicolumn{3}{|c|}{$\begin{array}{l}\text { Serious events detected on noncontrast CT } \\
\text { at } 24 \mathrm{hr}-\text { no./total no. (\%) }\end{array}$} \\
\hline \multicolumn{3}{|l|}{ Parenchymal hemorrhage } \\
\hline Type 1 & $2 / 129(1.6)$ & $4 / 115(3.5)$ \\
\hline Type 2 & $6 / 129(4.7)$ & $1 / 115(0.9)$ \\
\hline Subarachnoid hemorrhage & $3 / 129(2.3)$ & $1 / 115(0.9)$ \\
\hline Subdural hemorrhage & 0 & $1 / 115(0.9)$ \\
\hline Intraventricular hemorrhage & $1 / 129(0.8)$ & 0 \\
\hline \multicolumn{3}{|l|}{ Other serious events - no. (\%) } \\
\hline Cerebral ischemia & $13(8.4)$ & $10(6.8)$ \\
\hline Malignant brain edema & $17(11.0)$ & $7(4.8)$ \\
\hline Cardiac ischemia & $3(1.9)$ & $2(1.4)$ \\
\hline Pneumonia & $30(19.5)$ & $26(17.8)$ \\
\hline Other infection & $7(4.5)$ & $6(4.1)$ \\
\hline Extracranial hemorrhage & $5(3.2)$ & $2(1.4)$ \\
\hline Procedural complication & $5(3.2)$ & 0 \\
\hline Other complication & $20(13.0)$ & $25(17.1)$ \\
\hline
\end{tabular}

* A patient may have had more than one serious adverse event.

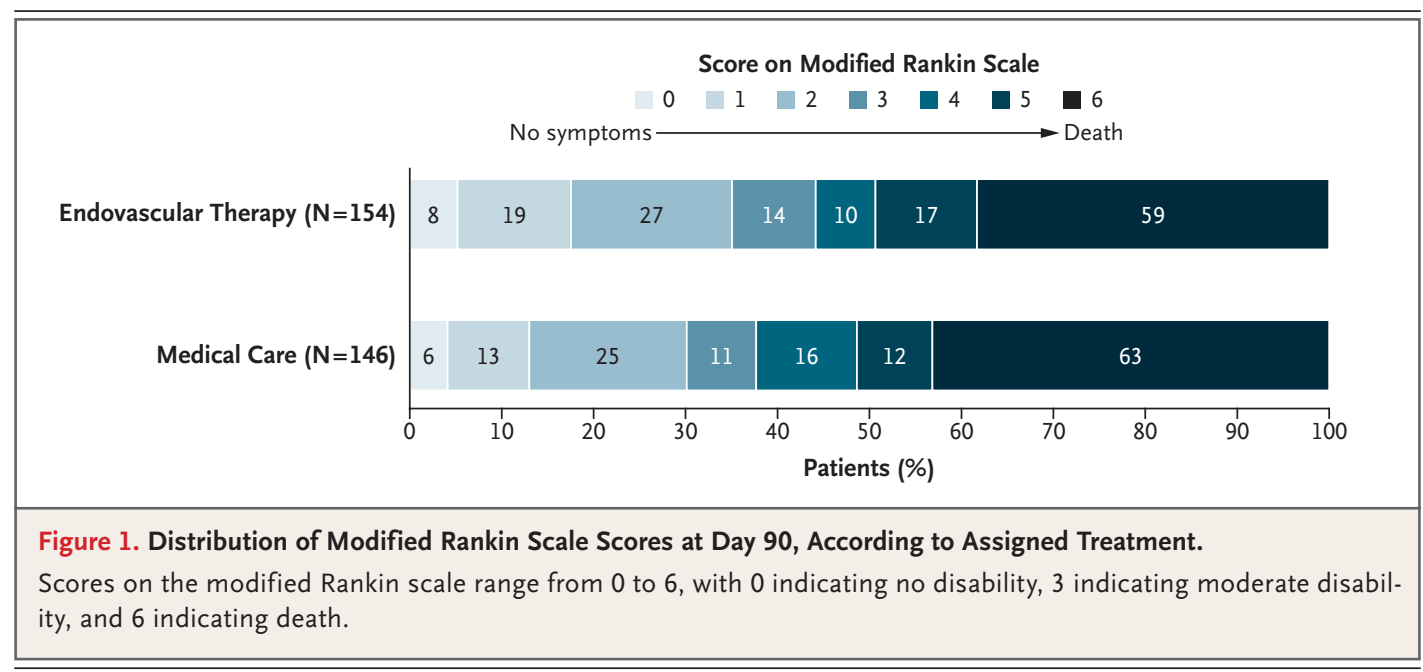




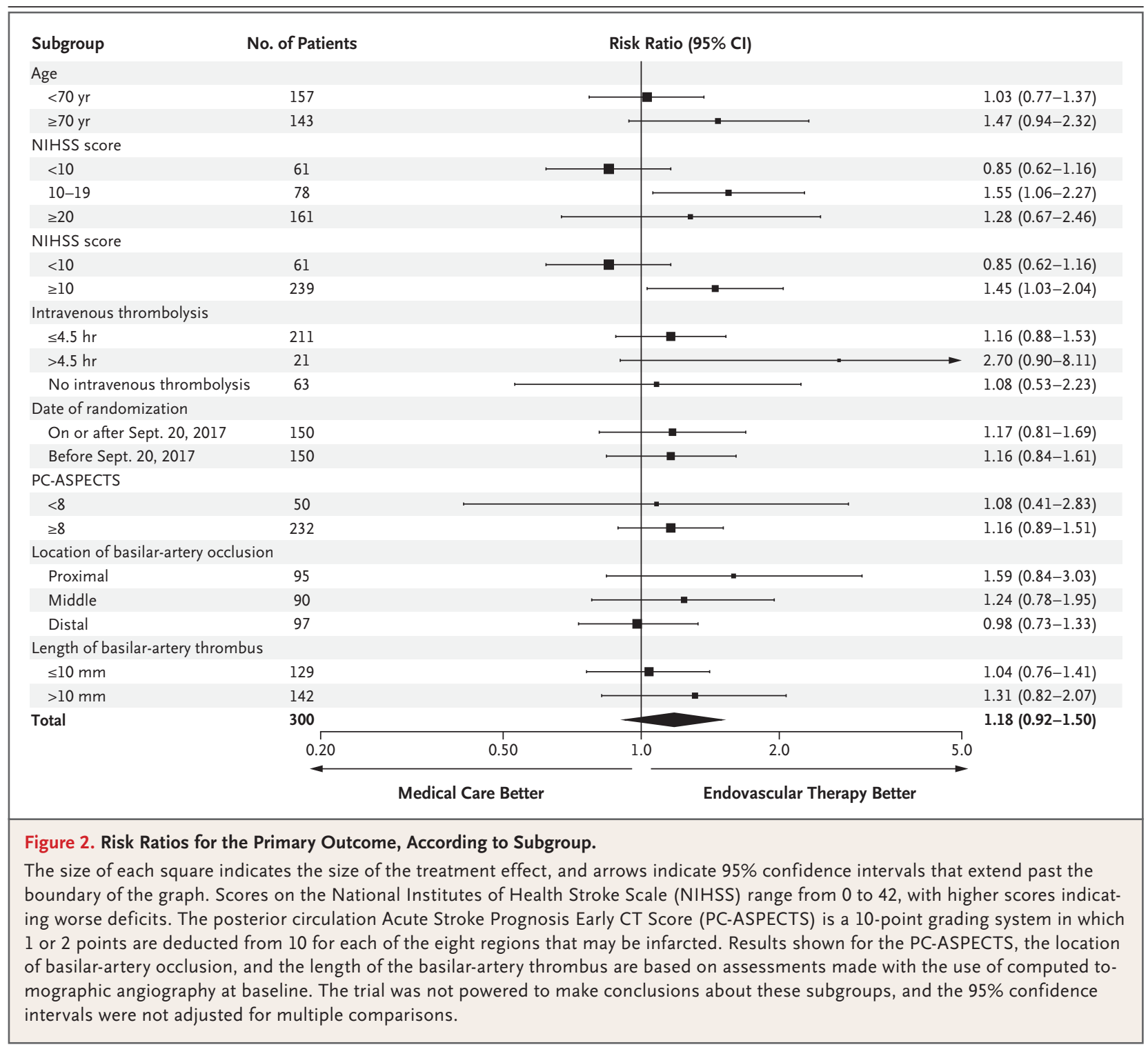

vascular Treatment versus Standard Medical Treatment for Vertebrobasilar Artery Occlusion (BEST) trial, ${ }^{19}$ was discontinued after the inclusion of 131 patients, before its intended enrollment, because of poor recruitment and a high rate of crossover from the medical care group to the endovascular therapy group. This suggests that, as in our multicenter trial, in which 300 patients were enrolled over a period of 8 years, recruitment of patients for trials of treatment for basilar-artery occlusion is difficult. The ongoing Basilar Artery Occlusion Chinese Endovascular (BAOCHE) trial (ClinicalTrials.gov number, NCT02737189), which is assessing the efficacy of endovascular therapy more than 6 hours after symptom onset in patients with basilar-artery occlusion, may inform the results of the current trial.

The limitations of our trial include data from screening logs showing that $29.2 \%$ of eligible patients were treated outside the trial and that $79.0 \%$ of these patients received endovascular therapy, a factor that may have introduced bias in the enrolled population. In addition, $5 \%$ of the patients in the medical treatment group crossed over to the endovascular group. We did not use advanced imaging, such as CT perfusion, for patient selection. There was an imbalance of patients 
with atrial fibrillation in the two treatment groups, but adjustment for this imbalance did not substantially alter effect size between the groups. The NIHSS, which was used for stratification in randomization, is less sensitive to symptoms of posterior-circulation stroke than to those of anterior-circulation stroke. Finally, because recruitment was lower than anticipated, our trial was underpowered for some analyses, including subgroup analyses.

In patients with basilar-artery occlusion, endovascular therapy and medical therapy were not significantly different with respect to a favorable functional outcome, but the results of our trial could not exclude a benefit of endovascular intervention. Data from larger trials are needed to determine the efficacy and safety of endovascular therapy in patients with basilar-artery occlusion.

This article is dedicated to Cristanne Wijman.

Supported by the Dutch Heart Foundation, the Swiss Heart Foundation, the São Paulo Research Foundation, the National Council for Scientific and Technological Development in Brazil, the University Medical Center Utrecht, and St. Antonius Hospital Nieuwegein.

Dr. Mont'Alverne reports receiving grant support and consulting fees from Balt USA, Medtronic, MicroVention, Penumbra, and Stryker; Dr. de Carvalho, receiving lecture fees, consulting fees, and travel support from Boehringer Ingelheim; Dr. Lima, receiving lecture fees from Boehringer Ingelheim; Dr. Dippel, receiving grant support, paid to Erasmus University Medical Center, from Cerenovus, Medtronic USA, Penumbra, Stryker European Operations, and Thrombolytic Science; Dr. van der Lugt, receiving grant support, paid to Erasmus University Medical Center, from Cerenovus, Health-Holland Top Sector Life Scienc- es and Health, Medtronic USA, Penumbra, Stryker, and Thrombolytic Science; Dr. van Zwam, receiving grant support, paid to Cardiovascular Research Institute Maastricht, from Johnson \& Johnson International and Stryker; Dr. Majoie, receiving grant support, paid to Amsterdam University Medical Center, from Stryker, and owning stock in Nico.lab; Dr. Gerber, receiving lecture fees from Penumbra; Dr. Mazighi, receiving consulting fees from Acticor Biotech, Air Liquide, Amgen, and Boehringer Ingelheim; Dr. Zini, receiving lecture fees from Johnson \& Johnson Health Care Systems and lecture fees and advisory board fees from Stryker; Dr. Martins, receiving lecture fees from Bayer, Medtronic, Penumbra, and Pfizer and lecture fees and advisory board fees from Boehringer Ingelheim; Dr. Nolte, receiving lecture fees from Abbott Canada, Bristol Myers Squibb, Pfizer, Portola Pharmaceuticals, and W.L. Gore and Associates and lecture fees and consulting fees from Boehringer Ingelheim; Dr. Abud, receiving lecture fees from Stryker; Dr. Yoo, receiving grant support, paid to Mercy Health-St. Vincent Medical Center, and steering committee fees from Johnson \& Johnson Medical Devices and Diagnostics Group-Latin America, grant support, paid to Mercy Health-St. Vincent Medical Center, from Medtronic and Stryker, grant support, paid to Mercy Health-St. Vincent Medical Center, and consulting fees from Penumbra, and owning stock in Insera Therapeutics; Dr. van Doormaal, receiving consulting fees from Stryker; Dr. Emmer, receiving grant support, paid to Amsterdam University Medical Center, from Nico.lab; Dr. Michel, receiving consulting fees, paid to his institution, from Medtronic and steering committee fees, paid to his institution, from Penumbra; Dr. Audebert, receiving grant support, advisory board fees, and lecture fees from Bayer, Boehringer Ingelheim, Bristol Myers Squibb, and Pfizer, consulting fees from Bayer Healthcare, fees for serving on a data and safety monitoring board from Novo Nordisk, and grant support and advisory board fees from Sanofi; and Dr. Pontes-Neto, receiving lecture fees from Bayer, Boehringer Ingelheim, Medtronic, and Pfizer. No other potential conflict of interest relevant to this article was reported.

Disclosure forms provided by the authors are available with the full text of this article at NEJM.org.

A data sharing statement provided by the authors is available with the full text of this article at NEJM.org.

\section{APPENDIX}

The authors' full names and academic degrees are as follows: Lucianne C.M. Langezaal, M.D., Erik J.R.J. van der Hoeven, M.D., Ph.D., Francisco J.A. Mont'Alverne, M.D., Ph.D., João J.F. de Carvalho, M.D., Fabrício O. Lima, M.D., Ph.D., Diederik W.J. Dippel, M.D., Ph.D., Aad van der Lugt, M.D., Ph.D., Rob T.H. Lo, M.D., Ph.D., Jelis Boiten, M.D., Ph.D., Geert J. Lycklama à Nijeholt, M.D., Ph.D., Julie Staals, M.D., Ph.D., Wim H. van Zwam, M.D., Ph.D., Paul J. Nederkoorn, M.D., Ph.D., Charles B.L.M. Majoie, M.D., Ph.D., Johannes C. Gerber, M.D., Mikael Mazighi, M.D., Ph.D., Michel Piotin, M.D., Ph.D., Andrea Zini, M.D., Stefano Vallone, M.D., Jeannette Hofmeijer, M.D., Ph.D., Sheila O. Martins, M.D., Ph.D., Christian H. Nolte, M.D., Ph.D., Kristina Szabo, M.D., Ph.D., Francisco A. Dias, M.D., Ph.D., Daniel G. Abud, M.D., Ph.D., Marieke J.H. Wermer, M.D., Ph.D., Michel J.M. Remmers, M.D., Hauke Schneider, M.D., Ph.D., Christina M. Rueckert, M.D., Karlijn F. de Laat, M.D., Ph.D., Albert J. Yoo, M.D., Ph.D., Pieter-Jan van Doormaal, M.D., Adriaan C.G.M. van Es, M.D., Ph.D., Bart J. Emmer, M.D., Ph.D., Patrik Michel, M.D., Ph.D., Volker Puetz, M.D., Heinrich J. Audebert, M.D., Ph.D., Octavio M. Pontes-Neto, M.D., Ph.D., Jan-Albert Vos, M.D., Ph.D., L. Jaap Kappelle, M.D., Ph.D., Ale Algra, M.D., Ph.D., and Wouter J. Schonewille, M.D., Ph.D.

The authors' affiliations are as follows: the Departments of Radiology (L.C.M.L., E.J.R.J.H., J.-A.V.) and Neurology (W.J.S.), St. Antonius Hospital, Nieuwegein, the Departments of Neurology (D.W.J.D.) and Radiology and Nuclear Medicine (A.L., P.-J.D.), Erasmus University Medical Center, Rotterdam, the Departments of Radiology (R.T.H.L.) and Neurology (L.J.K.) and the Department of Neurology and Neurosurgery, Brain Center, and the Julius Center for Health Sciences and Primary Care (A.A.), University Medical Center Utrecht, and Utrecht University (A.A.), Utrecht, the Departments of Neurology (J.B.) and Radiology (G.J.L.N.), Haaglanden Medical Center, and the Department of Neurology, Haga Hospital (K.F.L.), The Hague, the Departments of Neurology (J.S.) and Radiology and Nuclear Medicine (W.H.Z.), Cardiovascular Research Institute Maastricht (CARIM), Maastricht University Medical Center, Maastricht, the Departments of Neurology (P.J.N.) and Radiology (C.B.L.M.M., B.J.E.), Amsterdam University Medical Center, Amsterdam, the Department of Neurology, Rijnstate Hospital, Arnhem, and the Department of Clinical Neurophysiology, University of Twente, Enschede (J.H.), the Departments of Neurology (M.J.H.W.) and Radiology (A.C.G.M.E.), Leiden University Medical Center, Leiden, and the Department of Neurology, Amphia Hospital, Breda (M.J.M.R.) - all in the Netherlands; the Interventional Neuroradiology Service (F.J.A.M.) and the Neurology Service (J.J.F.C., F.O.L.), Hospital Geral de Fortaleza, Fortaleza, the Department of Neurology, Federal University of Rio Grande do Sul and Hospital de Clinicas de Porto Alegre, Porto Alegre (S.O.M.), and the Stroke Service, Neurology Division, Department of Neuroscience and Behavioral Sciences (F.A.D., O.M.P.-N.), and the Interventional Neuroradiology Service, 
Department of Medical Imaging, Hematology and Oncology (D.G.A.), Ribeirão Preto Medical School, University of São Paulo, São Paulo - all in Brazil; the Institute of Neuroradiology (J.C.G.) and the Department of Neurology (V.P.), Dresden Neurovascular Center, University Hospital Carl Gustav Carus, and Medizinische Fakultät Carl Gustav Carus (H.S.), Technische Universität Dresden, Dresden, the Department of Neurology and Center for Stroke Research, Charité Universitätsmedizin Berlin, Berlin (C.H.N., H.J.A.), the Department of Neurology, University Medical Center Mannheim, Medical Faculty Mannheim, Heidelberg University, Mannheim (K.S.), the Department of Neurology, University Hospital Augsburg, Augsburg (H.S.), and the Department of Neurology, Oberschwabenklinik, Ravensburg (C.M.R.) - all in Germany; Rothschild Foundation Hospital, INSERM Unité 1148, University of Paris, Paris (M.M., M.P.); the IRCCS Istituto delle Scienze Neurologiche di Bologna, Department of Neurology and Stroke Center, Maggiore Hospital, Bologna (A.Z.), and the Neuroradiology Unit, Department of Neuroscience, Ospedale Civile S. Agostino-Estense, Modena University Hospital, Modena (S.V.) — both in Italy; the Texas Stroke Institute, Fort Worth (A.J.Y.); and the Stroke Center, Neurology Service, Lausanne University Hospital, Lausanne, Switzerland (P.M.).

REFERENCES

1. Meinel TR, Kaesmacher J, ChaloulosIakovidis $\mathrm{P}$, et al. Mechanical thrombectomy for basilar artery occlusion: efficacy, outcomes, and futile recanalization in comparison with the anterior circulation. J Neurointerv Surg 2019;11:1174-80.

2. Mattle HP, Arnold M, Lindsberg PJ, Schonewille WJ, Schroth G. Basilar artery occlusion. Lancet Neurol 2011;10:1002-14. 3. Zeumer H, Hacke W, Ringelstein EB. Local intraarterial thrombolysis in vertebrobasilar thromboembolic disease. AJNR Am J Neuroradiol 1983;4:401-4.

4. Hacke W, Zeumer H, Ferbert A, Brückmann $\mathrm{H}$, del Zoppo GJ. Intra-arterial thrombolytic therapy improves outcome in patients with acute vertebrobasilar occlusive disease. Stroke 1988;19:1216-22. 5. Macleod MR, Davis SM, Mitchell PJ, et al. Results of a multicentre, randomised controlled trial of intra-arterial urokinase in the treatment of acute posterior circulation ischaemic stroke. Cerebrovasc Dis 2005;20:12-7.

6. Lindsberg PJ, Mattle HP. Therapy of basilar artery occlusion: a systematic analysis comparing intra-arterial and intravenous thrombolysis. Stroke 2006;37: 922-8.

7. Schonewille WJ, Wijman CAC, Michel $\mathrm{P}$, et al. Treatment and outcomes of acute basilar artery occlusion in the Basilar Artery International Cooperation Study (BASICS): a prospective registry study. Lancet Neurol 2009;8:724-30.

8. Goyal M, Menon BK, van Zwam WH, et al. Endovascular thrombectomy after large-vessel ischaemic stroke: a metaanalysis of individual patient data from five randomised trials. Lancet 2016;387: 1723-31.

9. van der Hoeven EJRJ, Schonewille WJ, Vos JA, et al. The Basilar Artery International Cooperation Study (BASICS): study protocol for a randomised controlled trial. Trials 2013;14:200

10. von Kummer R, Broderick JP, Campbell $\mathrm{BCV}$, et al. The Heidelberg Bleeding Classification; classification of bleeding events after ischemic stroke and reperfusion therapy. Stroke 2015;46:2981-6.

11. Puetz V, Sylaja PN, Coutts SB, et al. Extent of hypoattenuation on CT angiog raphy source images predicts functional outcome in patients with basilar artery occlusion. Stroke 2008;39:2485-90.

12. Berkhemer OA, Fransen PSS, Beumer D, et al. A randomized trial of intraarterial treatment for acute ischemic stroke. N Engl J Med 2015;372:11-20.

13. Saver JL, Goyal M, Bonafe A, et al. Stent-retriever thrombectomy after intra- venous t-PA vs. t-PA alone in stroke. $\mathrm{N}$ Engl J Med 2015;372:2285-95.

14. Jovin TG, Chamorro A, Cobo E, et al. Thrombectomy within 8 hours after symptom onset in ischemic stroke. $\mathrm{N}$ Engl J Med 2015;372:2296-306.

15. Campbell BCV, Mitchell PJ, Kleinig TJ, et al. Endovascular therapy for ischemic stroke with perfusion-imaging selection. N Engl J Med 2015;372:1009-18.

16. Mocco J, Zaidat OO, von Kummer R, et al. Aspiration thrombectomy after intravenous alteplase versus intravenous alteplase alone. Stroke 2016;47:2331-8.

17. Knol MJ, Le Cessie S, Algra A, Vandenbroucke JP, Groenwold RHH. Overestimation of risk ratios by odds ratios in trials and cohort studies: alternatives to logistic regression. CMAJ 2012;184:895-9. 18. Reinink $H$, Jüttler E, Hacke W, et al. Surgical decompression for space-occupying hemispheric infarction: a systematic review and individual patient meta-analysis of randomized clinical trials. JAMA Neurol 2021;78:208-16.

19. Liu X, Dai Q, Ye R, et al. Endovascular treatment versus standard medical treatment for vertebrobasilar artery occlusion (BEST): an open-label, randomised controlled trial. Lancet Neurol 2020;19:115-22. Copyright @ 2021 Massachusetts Medical Society. 